\title{
Importance of Endothelium-derived Hyperpolarizing Factor in Human Arteries
}

\author{
Lemmy Urakami-Harasawa, Hiroaki Shimokawa, Mikio Nakashima, ${ }^{*}$ Kensuke Egashira, and Akira Takeshita \\ Research Institute of Angiocardiology and Cardiovascular Clinic, Kyushu University School of Medicine, Fukuoka, Japan; and *Surgical \\ Center, Saga Medical School, Saga, Japan
}

\begin{abstract}
The endothelium plays an important role in maintaining the vascular homeostasis by releasing vasodilator substances, including prostacyclin ( $\left.\mathrm{PGI}_{2}\right)$, nitric oxide (NO), and endothelium-derived hyperpolarizing factor (EDHF). Although the former two substances have been investigated extensively, the importance of EDHF still remains unclear, especially in human arteries. Thus we tested our hypothesis that EDHF plays an important role in human arteries, particularly with reference to the effect of vessel size, its vasodilating mechanism, and the influences of risk factors for atherosclerosis. Isometric tension and membrane potentials were recorded in isolated human gastroepiploic arteries and distal microvessels (100-150 $\mu \mathrm{m}$ in diameter). The contribution of $\mathrm{PGI}_{2}$, NO, and EDHF to endothelium-dependent relaxations was analyzed by inhibitory effects of indomethacin, $N^{\mathrm{G}}$-nitro$\mathrm{L}$-arginine, and $\mathrm{KCl}$, respectively. The nature of and hyperpolarizing mechanism by EDHF were examined by the inhibitory effects of inhibitors of cytochrome $\mathrm{P} 450$ pathway and of various $K$ channels. The effects of atherosclerosis risk factors on EDHF-mediated relaxations were also analyzed. The results showed that $(a)$ the contribution of EDHF to endothelium-dependent relaxations is significantly larger in microvessels than in large arteries; $(b)$ the nature of EDHF may not be a product of cytochrome $\mathbf{P 4 5 0}$ pathway, while EDHF-induced hyperpolarization is partially mediated by calcium-activated $\mathrm{K}$ channels; and (c) aging and hypercholesterolemia significantly impair EDHF-mediated relaxations. These results demonstrate that EDHF also plays an important role in human arteries. (J. Clin. Invest. 1997. 100: 2793-2799.) Key words: endothelium-dependent relaxation • endothelium-derived hyperpolarizing factor • potassium channel $\bullet$ nitric oxide $\bullet$ atherosclerosis
\end{abstract}

\section{Introduction}

Animal studies have shown that endothelium-dependent relaxation is achieved by combined vasodilator effects of endo-

This work was presented in part at the annual scientific meeting of the American Heart Association, held in New Orleans, LA, 1996.

Address correspondence to Hiroaki Shimokawa, M.D., The Research Institute of Angiocardiology and Cardiovascular Clinic, Kyushu University School of Medicine, 3-1-1 Maidashi, Higashi-ku, Fukuoka 812-82, Japan. Phone: 81-92-642-5360; FAX: 82-92-6425375; E-mail: shimo@cardiol.med.kyushu-u.ac.jp

Received for publication 9 June 1997 and accepted in revised form 30 September 1997.

J. Clin. Invest.

(C) The American Society for Clinical Investigation, Inc. 0021-9738/97/12/2793/07 \$2.00

Volume 100, Number 11, December 1997, 2793-2799

http://www.jci.org thelium-derived prostacyclin $\left(\mathrm{PGI}_{2}\right),{ }^{1}$ nitric oxide (NO), and endothelium-derived hyperpolarizing factor (EDHF) (1-5). While the roles of the former two substances have been investigated extensively in both animals and humans (1-5), the importance of EDHF still remains unclear, especially in humans. Presently, only three studies have been reported regarding EDHF in human arteries. Nakashima et al. demonstrated, with electrophysiological data, that EDHF exists in human arteries (the coronary artery) excised from the failing human hearts obtained at heart transplantation (6). Subsequently, two other groups reported the endothelium-dependent relaxations resistant to the blockade of $\mathrm{PGI}_{2}$ or $\mathrm{NO}$ in human omental microvessels, but without electrophysiological data $(7,8)$.

We have shown recently that the importance of EDHF increases as the vessel size decreases in rat mesenteric arteries (9). Recent animal studies have suggested that EDHF may be arachidonic acid metabolites by cytochrome P450 monooxygenase (10-12), and that the hyperpolarizing mechanism by EDHF is mediated by $\mathrm{K}$ channels on the vascular smooth muscle (11-16). In addition, animal studies have shown that the EDHF-mediated relaxation is impaired by hypertension (17, 18), hypercholesterolemia (13, 19-21), diabetes mellitus (22), and aging $(17,23)$. However, all these points remain unclear in human arteries.

Thus, this study was designed to test our hypothesis that EDHF plays an important role in human arteries, particularly with reference to the effect of vessel size, its nature and vasodilating mechanism, and the influence of risk factors for atherosclerosis.

\section{Methods}

Study subjects. 77 patients (40 men and 37 women) who underwent gastrectomy operation were included in this study. Their ages ranged from 30 to $88 \mathrm{yr}$ (mean, $59 \mathrm{yr}$ ). Each patient was evaluated by physical examination and laboratory tests before the surgical operation for evaluation of atherosclerosis risk factors. The definition of hypercholesterolemia was $>220 \mathrm{mg} / \mathrm{dl}$ serum cholesterol and/or medication with cholesterol lowering agent. Diabetes mellitus was diagnosed when the fasting serum glucose level was $>120 \mathrm{mg} / \mathrm{dl}$ and/or when antidiabetes medication was taken. A patient was considered to have hypertension when he/she was taking antihypertensive agents or when his/her systolic blood pressure $>140 \mathrm{mmHg}$ or diastolic pressure $>90 \mathrm{mmHg}$ on at least three different occasions before the surgical operation. Risk factors for atherosclerosis examined in this study included age, hypercholesterolemia, diabetes mellitus, hypertension, and smoking.

The mean cholesterol level of the patients categorized to the hypercholesterolemia group was $207 \pm 9 \mathrm{mg} / \mathrm{dl}(n=22)$, and that categorized to the nonhypercholesterolemia group was $168 \pm 4 \mathrm{mg} / \mathrm{dl}(n=55$, $P<0.001)$. The mean blood glucose level of the patients categorized

1. Abbreviations used in this paper: ChTx, charybdotoxin; EDHF, endothelium-derived hyperpolarizing factor; IbTx, iberiotoxin; Kca, calcium-activated K channels; L-NNA, $N^{\mathrm{G}}$-nitro-L-arginine; NO, nitric oxide; $\mathrm{PGI}_{2}$, prostacyclin; TBA, tetrabutylammonium. 
to the diabetes group was $129 \pm 16 \mathrm{mg} / \mathrm{dl}(n=15)$, and that categorized to the nondiabetes group was $87 \pm 1 \mathrm{mg} / \mathrm{dl}(n=62, P<0.001)$. The mean blood pressure of the patients categorized to the hypertension group was $99 \pm 2 \mathrm{mmHg}(n=25)$, and that categorized to the nonhypertension group was $88 \pm 1 \mathrm{mmHg}(n=52, P<0.001)$.

This study was approved by the human research committee of the Institute. Since the arteries were classified as surgical specimens, their use was exempted by the institutional review board from required patient consent.

Human arteries. During the gastrectomy operation, right gastroepiploic arteries and nets were carefully removed.

The obtained vessels were carried in cold Krebs solution. Proximal and distal gastroepiploic arteries were cleaned carefully of adherent perivascular connective tissue under a microscope (9). Proximal arteries were cut into 5-mm rings, and their distal microvessels were cut into 3-mm rings.

Organ chamber experiments. All experiments were performed in $37^{\circ} \mathrm{C}$ Krebs solution, which was bubbled by $95 \% \mathrm{O}_{2}$ and $5 \% \mathrm{CO}_{2}$. The rings of arteries were mounted in organ chambers filled with Krebs solution ( $20 \mathrm{ml}$ of capacity for proximal arteries, and $2 \mathrm{ml}$ of capacity for distal arteries) (Medical Supply Co., Osaka, Japan) in two stainless steel wires; one was anchored and the other was connected to the force transducer (Nihon Kohden, Tokyo, Japan) (9). An optimal resting tension of $2 \mathrm{~g}$ was applied to the rings from proximal arteries, and $100 \mathrm{mg}$ to those from distal arteries, respectively (9). The rings were allowed to equilibrate for at least $60 \mathrm{~min}$ before the start of the experiments. The rings were repeatedly challenged with $60 \mathrm{mmol} /$ liter $\mathrm{KCl}$ until the $\mathrm{KCl}$-induced contractions reached a constant value (9).

In arteries, the relaxation responses to bradykinin or acetylcholine were examined during a contraction to $\mathrm{PGF}_{2 \alpha}$. To examine the contribution of endothelium-dependent relaxation, relaxations were examined under the following four conditions (9); $(a)$ in the absence of any drug (control response); $(b)$ in the presence of indomethacin $\left(10^{-5} \mathrm{~mol} /\right.$ liter) (to inhibit $\mathrm{PGI}_{2}$ production); $(c)$ in the presence of indomethacin and $N^{\mathrm{G}}$-nitro-L-arginine (L-NNA) $\left(10^{-4} \mathrm{~mol} / \mathrm{liter}\right)$ (to inhibit the production of both $\mathrm{PGI}_{2}$ and $\left.\mathrm{NO}\right)$; and $(d)$ in the presence of indomethacin, L-NNA, and $\mathrm{KCl}(20-40 \mathrm{mmol} / \mathrm{liter})$ (to inhibit the production of $\mathrm{PGI}_{2}, \mathrm{NO}$, and EDHF). In a preliminary study, we confirmed that $10^{-4} \mathrm{~mol} / \mathrm{liter}$ of L-NNA was sufficient to completely suppress the increase in tissue cGMP levels caused by bradykinin $(n=3$, data not shown). The relaxations under the four conditions were examined in parallel in four different rings with intact endothelium.

The following inhibitors were used to examine the nature of EDHF and the mechanism of EDHF-induced relaxation and hyperpolarization: SKF 525a (an inhibitor of cytochrome P450 monooxygenase $3 \times 10^{-6} \mathrm{~mol} /$ liter) (12), tetrabutylammonium (TBA, a nonselective inhibitor of $\mathrm{K}$ channels $10^{-2} \mathrm{~mol} / \mathrm{liter}$ ), charybdotoxin (ChTx, an inhibitor of both large and intermediate conductance calcium-activated $\mathrm{K}$ channels [Kca] $5 \times 10^{-8} \mathrm{~mol} /$ liter) (24), iberiotoxin (IbTx, an inhibitor of large conductance Kca $3 \times 10^{-8} \mathrm{~mol} / \mathrm{liter}$ ) (25), and apamin (an inhibitor of small conductance Kca $10^{-6} \mathrm{~mol} /$ liter) (15). These examinations were performed only in large arteries for the following reasons. First, it was easier to record membrane potentials in large arteries than in microvessels. Second, large arteries were more frequently available than microvessels. Third, the number of chambers for microvessels was limited.

All responses were displayed on a pen writing recorder (model 3056; Yokogawa Hokushin Electric, Fujisawa, Japan).

Membrane potential recording. Transverse strips were prepared by cutting along the longitudinal axis of the rings. The rings of proximal arteries were opened longitudinally and placed in an experimental chamber (capacity, $2 \mathrm{ml}$ ) with the endothelial layer up (9). The rings of microvessels were placed in the chamber without an opening (9). The tissue specimens were superfused with $37^{\circ} \mathrm{C}$ Krebs solution bubbled with $95 \% \mathrm{O}_{2}$ and $5 \% \mathrm{CO}_{2}(\mathrm{pH} 7.3-7.4)$ at a rate of $3 \mathrm{ml} / \mathrm{min}$ and then were allowed to equilibrate for at least $60 \mathrm{~min}$ before the start of the recordings. The membrane potential recording was de- scribed in detail previously $(6,9)$. Briefly, glass capillary microelectrodes filled with $3 \mathrm{~mol} / \mathrm{liter} \mathrm{KCl}$ (tip resistances of 50-80 M and tip potentials $<4 \mathrm{mV}$ ) were impaled into the smooth muscle cell from the endothelial side in the strips of large arteries, and from the adventitial side in the strips of microvessels (9). The criteria for a successful impalement consisted of an abrupt drop in the voltage on entry of the microelectrode into the cell, a stable membrane potential for at least $2 \mathrm{~min}$, and a sharp return to zero on withdrawal of the electrode (9). Changes in the membrane potentials produced by bradykinin were measured from the continuous recordings. In a preliminary study, we confirmed that $40 \mathrm{mmol} /$ liter $\mathrm{KCl}$ completely inhibits the bradykinininduced hyperpolarizations in human arteries (data not shown). The electrical responses were monitored on an oscilloscope (model VC11; Nihon Kohden, Tokyo, Japan) and recorded on a pen recorder (model RJG-4002; Nihon Kohden Co.).

Solutions and drugs. The ionic millimolar composition of Krebs solution was as follows: $134.7 \mathrm{Na}^{+}, 5.9 \mathrm{~K}^{+}, 1.2 \mathrm{Mg}^{+}, 2.5 \mathrm{Ca}^{+}, 1.2$ $\mathrm{H}_{2} \mathrm{PO}_{4}^{-}, 15.5 \mathrm{HCO}_{3}^{-}, 137 \mathrm{Cl}^{-}$, and 11.5 glucose. The concentrations of $\mathrm{K}^{+}$were modified by replacing $\mathrm{NaCl}$ with $\mathrm{KCl}$. The drugs used were acetylcholine chloride, bradykinin, indomethacin, L-NNA, ChTx, IbTx, apamin, and TBA (Sigma Chemical Co., St. Louis, MO). Indomethacin was dissolved in $10^{-2} \mathrm{~mol} /$ liter $\mathrm{Na}_{2} \mathrm{CO}_{3}$. Other drugs were dissolved in distilled water. The solvents used to dissolve the drugs did not, by themselves, affect the mechanical or electrical responses at their final bath concentrations.

Statistical analysis. The relative contribution of NO and EDHF to endothelium-dependent relaxations was determined as follows: the contribution of NO expressed by the relaxation between the responses in the presence of indomethacin and those in the presence of indomethacin and L-NNA, and the contribution of EDHF expressed by the relaxation between the responses in the presence of indomethacin and L-NNA and those in the presence of the two inhibitors and $\mathrm{KCl}(9)$.

Results are expressed as mean \pm SEM. The dose-response curves of relaxations and hyperpolarizations were analyzed by a two-way ANOVA followed by a Fisher's post-hoc test for multiple comparisons. Values $<0.05$ were considered to be statistically significant.

To determine which risk factor(s) would significantly influence the EDHF-mediated endothelium-dependent relaxations, we performed univariable analysis followed by multivariable analysis. In this analysis, the dependent variable was the percentage of relaxation as compared with the difference in force between the precontraction and after $40 \mathrm{mmol} /$ liter of $\mathrm{KCl}$ in the presence of indomethacin and L-NNA, while the independent variables were age and number of risk factors as continuous variables, and hypercholesterolemia, hypertension, diabetes mellitus, and smoking as dichotomous variables.

\section{Results}

Effect of vessel size. Fig. 1 shows relaxation responses to bradykinin and to acetylcholine under the four different conditions in large arteries and microvessels. The extent of the relaxations to acetylcholine was smaller than that to bradykinin in both sizes of human arteries. Indomethacin did not affect the bradykinin- or acetylcholine-induced relaxations in both sizes of arteries. In the presence of indomethacin and L-NNA, bradykinin-induced relaxations were suppressed significantly in large arteries but not in microvessels. In the presence of indomethacin, L-NNA, and $\mathrm{KCl}$, bradykinin-induced relaxations were largely inhibited in both sizes of human arteries. As in the case with bradykinin, combined treatment with indomethacin and L-NNA inhibited the acetylcholine-induced relaxations in large arteries but not in microvessels. Those acetylcholine-induced relaxations in microvessels were abolished by $\mathrm{KCl}$ in the presence of indomethacin and L-NNA.

The resting membrane potentials in rings without endothe- 

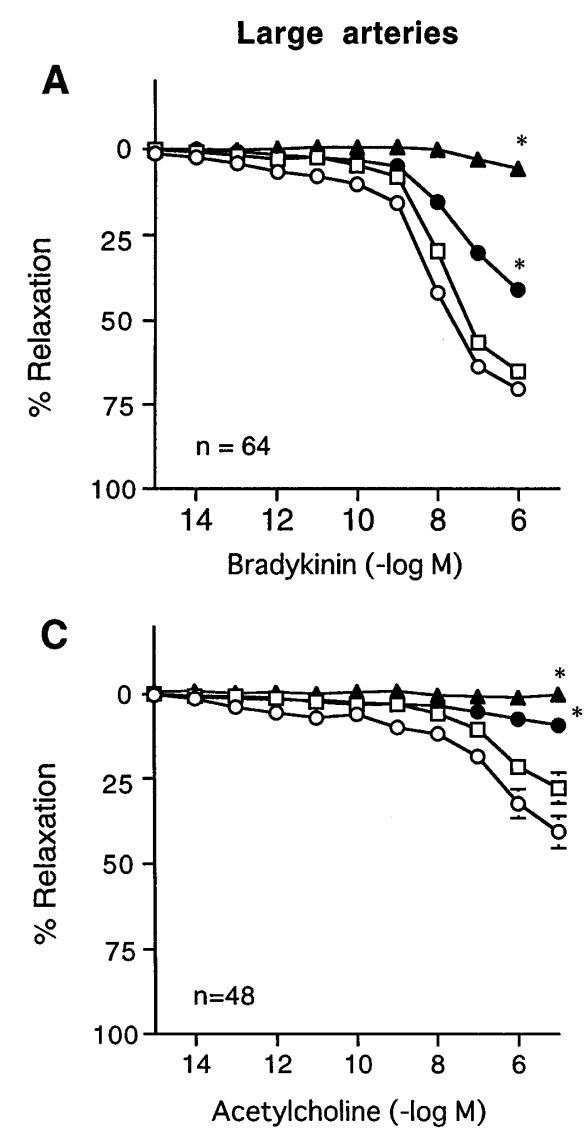
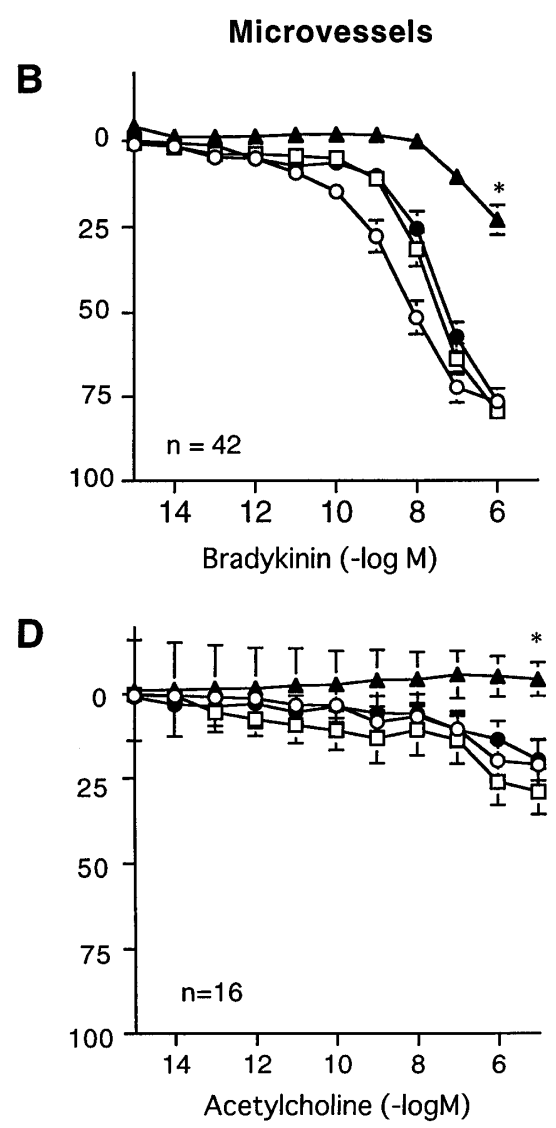

Figure 1. Endothelium-dependent relaxations to bradykinin ( $A$ and $B$ ) and acetylcholine $(C$ and $D)$ in human arteries. Results are presented as mean \pm SEM. $A$ and $C$ represent the result with large arteries, and $B$ and $D$ show the result with microvessels. Open circles, control; open squares, indomethacin $\left(10^{-5} \mathrm{~mol} / \mathrm{liter}\right)$; filled circles, indomethacin plus L-NNA $\left(10^{-4} \mathrm{~mol} / \mathrm{liter}\right)$; filled triangles, indomethacin plus L-NNA plus $\mathrm{KCl}$ (20-40 mmol/liter). *Difference in the maximal relaxations compared with those in the control responses $(P<0.0001$ vs. control). The relaxations to acetylcholine were smaller than those to bradykinin in both large arteries and microvessels. L-NNA in the presence of indomethacin reduced the relaxations in large arteries but not in microvessels. lium were significantly more negative in microvessels than in large arteries (Table I). This also was the case in rings with endothelium either after the treatment with indomethacin alone or with both indomethacin and L-NNA (Table I).

Bradykinin caused hyperpolarizations in both sizes of arteries, while the acetylcholine-induced hyperpolarizations were not evident (Fig. 2). The membrane potentials were significantly more negative in microvessels than in large arteries in all ranges of concentrations of both agonists (Fig. 2).

Nature of EDHF and mechanism for EDHF-mediated relaxations and hyperpolarizations. The effects of several inhibitors on the indomethacin- and L-NNA-resistant endothelium-dependent relaxations to bradykinin are shown in Fig. 3. The relaxations were not suppressed by SKF 525a, IbTx, or apamin, while ChTx partially reduced and TBA almost abolished the relaxations (Fig. 3). TBA also markedly inhibited both the resting membrane potentials and hyperpolarizations

Table I. Resting Membrane Potentials in Human Arteries

\begin{tabular}{lcc}
\hline & Large arteries & Microvessels \\
\hline Without endothelium & $-52.2 \pm 1.6(6)$ & $-65.3 \pm 2.0^{*}(5)$ \\
With endothelium & & \\
$\quad$ Indomethacin & $-54.8 \pm 1.4(6)$ & $-63.7 \pm 2.1 *(5)$ \\
$\quad$ Indomethacin + L-NNA & $-53.6 \pm 1.1(6)$ & $-64.6 \pm 1.8^{*}(5)$
\end{tabular}

Data are presented as mean $(\mathrm{mV}) \pm \mathrm{SEM}$. Number of animals is shown in parentheses. ${ }^{*} P<0.01$ vs. large arteries. to bradykinin, while ChTx significantly inhibited the hyperpolarizations to bradykinin (Fig. 4). Other inhibitors did not show any inhibitory effect (Fig. 4).

Influence of atherosclerosis risk factors. To examine the influence of atherosclerosis risk factors on EDHF-mediated responses, we used the results of the relaxations by EDHF caused by $10^{-6} \mathrm{~mol} /$ liter bradykinin as shown in Fig. 1 . Univariable analysis revealed that age, hypercholesterolemia, and the number of risk factors were significant univariable predictors, while other risk factors, such as hypertension, diabetes, or smoking, were not significant predictors (Table II and Fig. 5). Multivariable analysis using these significant univariable predictors further demonstrated that age and hypercholesterolemia were again significant predictors (Table II). The effect of age was equally noted in both men and women (data not shown).

\section{Discussion}

The novel findings of this study using human gastroepiploic arteries were that $(a)$ the role of EDHF is significantly greater in microvessels than in large arteries; $(b)$ the nature of EDHF may not be a product of cytochrome P450 pathway, while EDHF-induced hyperpolarization is mediated by $\mathrm{K}$ channels; and (c) aging and hypercholesterolemia significantly impair the EDHF-mediated relaxations. To our knowledge, this is the first study that demonstrated the importance of EDHF in human arteries.

EDHF in human arteries. EDHF-mediated endotheliumdependent relaxations have been documented repeatedly in 
A

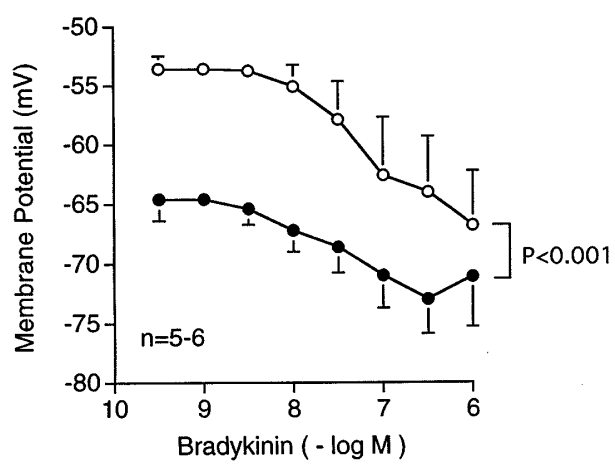

B

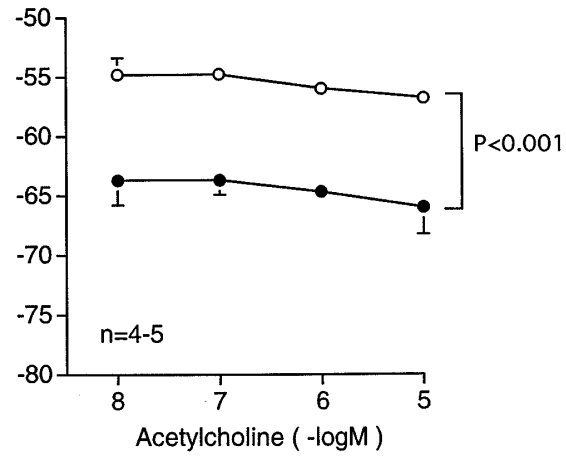

Figure 2. Endothelium-dependent hyperpolarizations to bradykinin $(A)$ and to acetylcholine $(B)$. Results are presented as mean \pm SEM. The membrane potentials were significantly deeper in microvessels (filled circles) than in large arteries (open circles). animals (1-5). However, the existence of EDHF in human arteries has been reported previously only in three studies. Nakashima et al. demonstrated the existence of EDHF in human arteries (6). However, since human coronary arteries obtained from failing hearts were used in the study, the reported results may not necessarily represent the aspects of EDHF in normal human arteries. In addition, microvessels were not examined in that study. In this study, we used gastroepiploic arteries and their microvessels from patients who underwent gastrectomy for gastric cancer without any chemotherapy. Therefore we consider that human arteries that we used in this study represent the normal conditions of EDHF. Subsequently, two other groups have demonstrated the endothelium-dependent relaxations (to bradykinin and substance $\mathrm{P}$ ) that were resistant to the blockade of $\mathrm{PGI}_{2}$ and $\mathrm{NO}$ in human omental microvessels $(7,8)$. However, in these studies no electrophysiological study was performed and thus it was unknown whether such relaxations were associated with endothelium-dependent hyperpolarizations. In this study, we demonstrated the EDHF-mediated hyperpolarizations and relaxations in different sizes of human arteries and attempted to elucidate the importance of EDHF in human arteries.

It is methodologically important to confirm that after inhibition of the production of $\mathrm{PGI}_{2}$ and $\mathrm{NO}, \mathrm{EDHF}$-mediated responses alone were examined in this study. First, we confirmed in a preliminary study that the present concentration of L-NNA was sufficient to completely suppress the bradykinin-induced increase in the vascular cGMP levels. Second, we confirmed that the resting membrane potentials and hyperpolarizations were not significantly modified by indomethacin or L-NNA, which indicates that endogenous $\mathrm{PGI}_{2}$ or $\mathrm{NO}$ itself does not cause hyperpolarization in human gastroepiploic arteries. This finding was consistent with our recent finding in rat mesenteric arteries (9).

Effect of vessel size. The degree of endothelium-dependent relaxations to bradykinin was comparable between large arteries and microvessels. However, the contribution of NO and EDHF to the relaxations was markedly dependent on the vessel size; in large arteries both NO and EDHF equally contributed, while in microvessels most of the relaxations were achieved by EDHF. These functional results well correlated with the results from our electrophysiological experiments, which showed that membrane potentials were significantly more negative at most of the concentrations of bradykinin in microvessels than in large arteries. The degree of hyperpolarizations to acetylcholine was smaller in both large arteries and microvessels, together with smaller relaxations to the muscarinic agonist.

Several mechanisms may be possible to explain the larger contribution of EDHF in microvessels than in large arteries in humans (9). First, microvessels may release more EDHF. Second, the vascular smooth muscle in microvessels may be more responsive to EDHF. Indeed, the difference in resting membrane potentials between large arteries and microvessels was
A

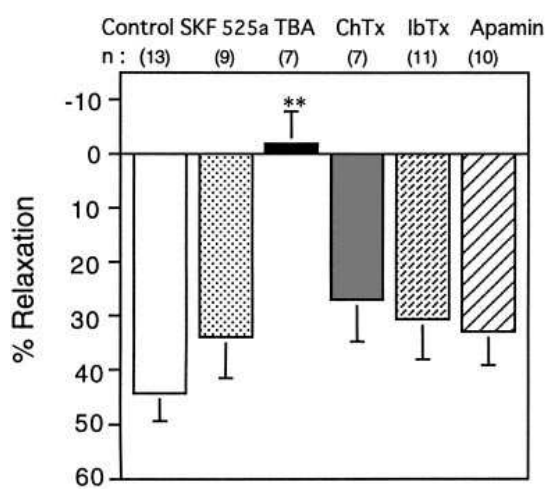

B Bradykinin $\left(10^{-6} \mathrm{M}\right)$

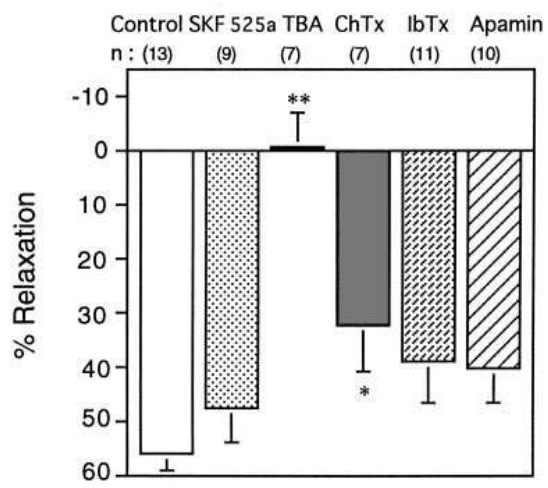

Figure 3. Effects of inhibitors on indomethacin/L-NNA-resistant endothelium-dependent relaxations to bradykinin in large human arteries. All responses were examined in the presence of $10^{-5} \mathrm{~mol} / \mathrm{liter}$ indomethacin and $10^{-4} \mathrm{~mol} / \mathrm{liter} \mathrm{L}-\mathrm{NNA}$. Results are presented as mean \pm SEM. $A$ shows responses to $10^{-7} \mathrm{~mol} /$ liter bradykinin; $B$ shows responses to $10^{-6} \mathrm{~mol} /$ liter bradykinin. TBA abolished the EDHFmediated relaxations, and ChTx significantly inhibited those relaxations (at $10^{-6} \mathrm{~mol} /$ liter), while other inhibitors showed no inhibitory effect on the relaxations. $* P<0.05$ vs. control; $* * P<0.01$ vs. control. 


\section{A. Resting membrane potential $(* \mathrm{P}<0.001)$}
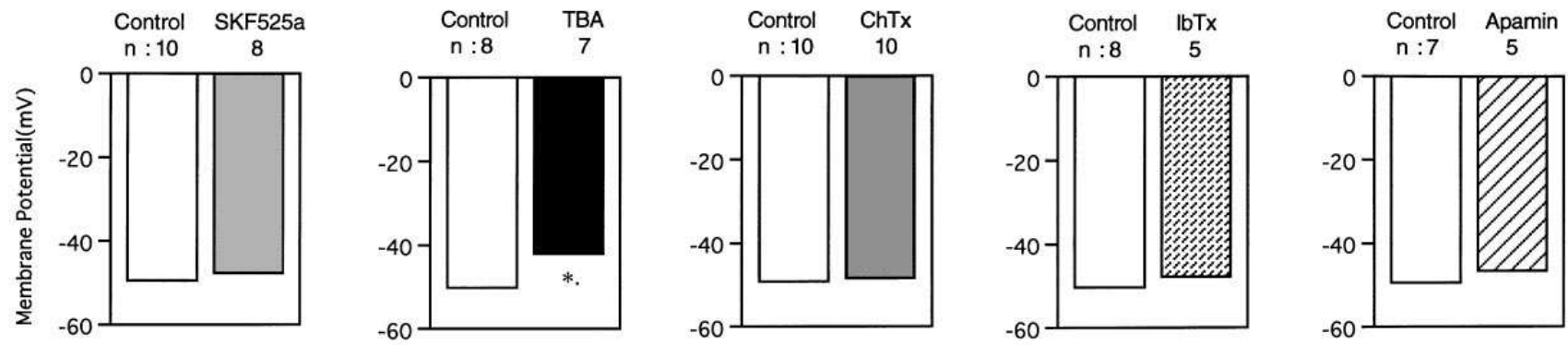

\section{B. Hyperpolarization to bradykinin $(* P<0.001)$}
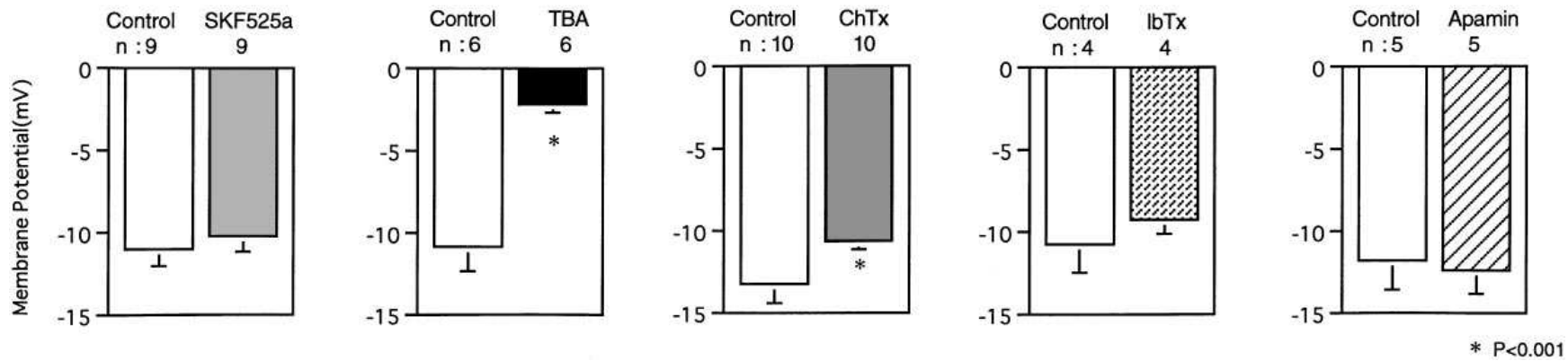

Figure 4. Resting membrane potential $(A)$ and endothelium-dependent hyperpolarization to bradykinin $(B)\left(10^{-6} \mathrm{~mol} /\right.$ liter $)$ in human arteries. All responses were examined in the presence of $10^{-5} \mathrm{~mol} /$ liter indomethacin and $10^{-4} \mathrm{~mol} / \mathrm{liter} \mathrm{L}-\mathrm{NNA}$. Results are presented as mean $\pm \mathrm{SEM}$. TBA significantly inhibited both the resting membrane potentials and the hyperpolarizations to bradykinin, while ChTx significantly inhibited the hyperpolarizations to bradykinin. Other inhibitors showed no inhibitory effect on the two electrophysiological parameters.

not affected by endothelium removal, or inhibition of the production of either $\mathrm{PGI}_{2}$ or $\mathrm{NO}$, which suggests that the difference in resting membrane potentials was determined primarily by the difference in smooth muscle properties. Third, EDHFinduced vascular relaxations may be determined by the combined effects of the resting membrane potentials and the agonist-induced hyperpolarizations. Indeed, the difference in the membrane potentials in response to bradykinin resulted primarily from the difference in the resting membrane potentials. Fourth, EDHF may more easily diffuse to the underlying vascular smooth muscle in microvessels than in large arteries, or EDHF may have too short of a half life to diffuse through large arterial smooth muscle layers.

We also have observed recently that the contribution of EDHF to endothelium-dependent relaxations is significantly larger in microvessels than in large arteries, not only in rat mesenteric arteries (9) but also in porcine and rabbit coronary arteries (26). Thus, the greater role of EDHF in microvessels than in large arteries may be the case in different vascular beds in different species.

Nature of EDHF in human arteries. The nature of EDHF has been suggested recently as an arachidonic acid metabolite of cytochrome P450 monooxygenase $(10-12,16)$. However, it should be noted that these experiments were done in bovine arteries. Weintraub et al. reported that the nature of acetyl- choline-induced EDHF in porcine coronary arteries may not be a metabolite of cyclooxygenase or cytochrome $\mathrm{P} 450$ monooxygenase (27). Corriu et al. reached the same conclusion with the guinea pig carotid artery (28). In the present study, bradykinin-induced relaxations and hyperpolarizations were not affected by cytochrome P450 inhibitor, SKF 525a. Although we used only one cytochrome P450 inhibitor, the concentration that we used was comparable to that Bauersachs et al. used in rat microvessels (12). Thus, we consider that there may be more than one EDHF, depending on the species examined, blood vessels tested, and agonists used, and that the nature of EDHF in human gastroepiploic arteries in response to bradykinin may not be an arachidonic acid metabolite of cytochrome $\mathrm{P} 450$ monooxygenase.

Mechanism for EDHF-mediated relaxation and hyperpolarization in human arteries. Previous studies demonstrated that the endothelium-dependent relaxations and hyperpolarizations in the presence of inhibitors of $\mathrm{PGI}_{2}$ and $\mathrm{NO}$ synthesis are markedly suppressed by K channel inhibitors $(10-16,28)$. However, no consensus has been made regarding the subtype of $\mathrm{K}$ channel that mediates EDHF-induced hyperpolarization. In this study, we used five different inhibitors of $\mathrm{K}$ channels ( $\mathrm{KCl}, \mathrm{TBA}$, apamin, IbTx, and ChTx) and demonstrated that $\mathrm{KCl}$, TBA, and ChTx exerted an inhibitory effect on the EDHF-induced hyperpolarizations and relaxations. Since 
Table II. Univariable and Multivariable Analysis of the Influence of Atherosclerosis Risk Factors on EDHF-mediated Response in Human Gastroepiploic Arteries

\begin{tabular}{|c|c|c|c|c|}
\hline \multicolumn{5}{|l|}{ Large arteries $(n=77)$} \\
\hline Univariable analysis & (yes/no) & Monovariable regression & $R$ & $P$ \\
\hline Age & & $y=88.3-0.7 x$ & 0.42 & $0.002 *$ \\
\hline Hypercholesterolemia & $(22 / 55)$ & $y=50.1-17 x$ & 0.34 & $0.003^{*}$ \\
\hline Hypertension & $(25 / 52)$ & $y=47.9-6 x$ & 0.12 & 0.29 \\
\hline Diabetes mellitus & $(15 / 62)$ & $y=46.2-2 x$ & 0.04 & 0.76 \\
\hline Smoking & $(42 / 35)$ & $y=47.2+2 x$ & 0.06 & 0.63 \\
\hline Number of risk factors & & $y=52.8-5 x$ & 0.26 & $0.02 *$ \\
\hline $\operatorname{Sex}(M / F)$ & $(41 / 36)$ & $y=48.4-5 x$ & 0.11 & 0.34 \\
\hline Multivariable analysis & $R=0.49, P=0.0001$ & Coefficient & $t$ & $P$ \\
\hline Age & & -0.7 & -3.2 & $0.002 *$ \\
\hline Hypercholesterolemia & & -11.8 & -2.3 & $0.046^{*}$ \\
\hline Number of risk factors & & 0.06 & 0.02 & 0.98 \\
\hline \multicolumn{5}{|l|}{ Microvessels $(n=42)$} \\
\hline Univariable analysis & (yes/no) & Monovariable regression & $R$ & $P$ \\
\hline Age & & $y=76.5+0.002 x$ & 0.001 & 0.99 \\
\hline Hypercholesterolemia & $(6 / 36)$ & $y=76.2+4 x$ & 0.06 & 0.69 \\
\hline Hypertension & $(13 / 29)$ & $y=74.8+4.1 x$ & 0.10 & 0.56 \\
\hline Diabetes mellitus & $(4 / 38)$ & $y=75.9+7 x$ & 0.11 & 0.50 \\
\hline Smoking & $(22 / 20)$ & $y=72.4+7 x$ & 0.06 & 0.63 \\
\hline Number of risk factors & & $y=70.3+5 x$ & 0.06 & 0.13 \\
\hline $\operatorname{Sex}(M / F)$ & $(27 / 16)$ & $y=48.4-5 x$ & 0.11 & 0.34 \\
\hline
\end{tabular}

IbTx is a pure inhibitor of large Kca and ChTx is an inhibitor of both large and intermediate Kca, these results suggest that intermediate Kca may be involved in the bradykinin-induced hyperpolarization and relaxation in human gastroepiploic arteries. However, the subtype of Kca in the EDHF-mediated relaxations in human arteries remains to be fully clarified in a future study.

Influence of atherosclerosis risk factors. Many studies demonstrated that atherosclerosis risk factors, such as hypercholesterolemia, hypertension, diabetes mellitus, and smoking, impair NO-mediated endothelium-dependent relaxations in both humans and animals (1-5). Several animal studies also suggested that EDHF-mediated endothelium-dependent responses could also be reduced by such factors, including hypertension (17, 18), hypercholesterolemia $(13,19-21)$, diabetes mellitus (22), and aging $(17,23)$. We also observed recently that estrogen augments both NO-mediated and non-NO-, non- $\mathrm{PGI}_{2}$-mediated (possibly EDHF-mediated) endothelium-dependent forearm vasodilation in postmenopausal women (29). However, the influence of those risk factors on the EDHF-mediated responses in humans remains to be elucidated. Our univariable and multivariable analyses showed that aging and hypercholesterolemia significantly reduced the EDHF-mediated relaxations in large arteries. Hypertension also tended to impair the

\section{A}

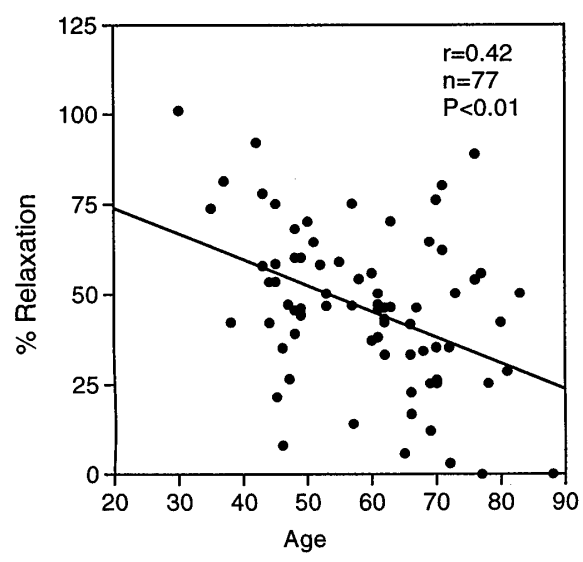

B

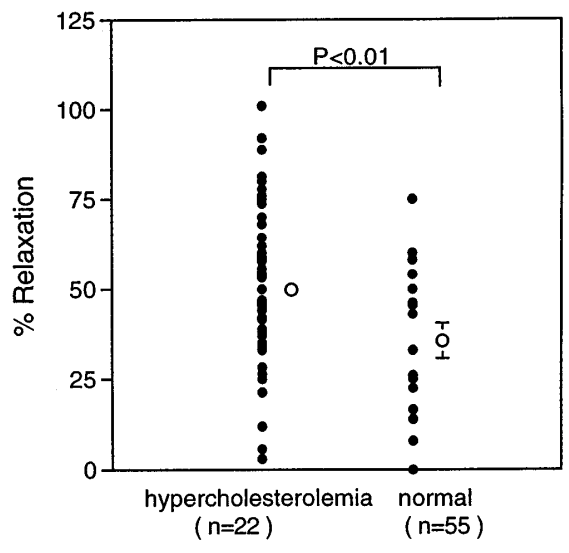

Figure 5. Influences of aging $(A)$ and of hypercholesterolemia $(B)$ on the EDHFmediated component of the bradykinininduced endothelium-dependent relaxations in human arteries. Vertical bars represent the absolute relaxation (\%) to bradykinin $\left(10^{-6} \mathrm{~mol} /\right.$ liter $)$ in the presence of indomethacin $\left(10^{-5} \mathrm{~mol} / \mathrm{liter}\right)$ and L-NNA $\left(10^{-4} \mathrm{~mol} /\right.$ liter $)$. Results are presented as mean \pm SEM in B. Hypercholesterolemia, patients with hypercholesterolemia; normal, patients without hypercholesterolemia (several points are overlapped). 
EDHF-mediated responses in large arteries. However, none of the risk factors significantly affected the EDHF-mediated responses in microvessels. The reason for the different influence of the risk factors on the EDHF-mediated responses between large arteries and microvessels remains to be examined.

In summary, we demonstrated that EDHF plays an important role in endothelium-dependent relaxations in human arteries, especially in microvessels. The nature of EDHF in human arteries may not be a metabolite of cytochrome P450 monooxygenase pathway, while the effect of EDHF is mediated in part by Kca channels. The EDHF-mediated relaxations are impaired in large arteries by aging and hypercholesterolemia, but fairly preserved in microvessels despite the presence of atherosclerosis risk factors. It is possible that EDHF contributes to maintaining the vascular homeostasis when NOmediated responses are impaired by atherosclerosis risk factors.

\section{Acknowledgments}

We thank Dr. Tanaka, Dr. Sato, and Dr. Kamei (The First Department of Surgery, Kyushu University School of Medicine), and Dr. Saku and T. Tanaka (The Department of Surgery, National Hospital Kyushu Medical Center) for cooperation in this study, Dr. H. Suzuki (Department of Physiology, Nagoya City University) for valuable comments on our work, and T. Takebe, E. Gunshima, and E. Tajima for technical assistance.

This work was supported in part by a grant-in-aid from Japan's Ministry of Education, Science, Sports, and Culture, Tokyo, Japan.

\section{References}

1. Vanhoutte, P.M., editor. 1996. Endothelium-derived Hyperpolarizing Factor. Harwood Academic Publishers, Amsterdam.

2. Lüscher, T.F., and P.M. Vanhoutte. 1990. The Endothelium: Modulator of Cardiovascular Function. CRC Press, Boca Raton, FL.

3. Cohen, R.A., and P.M. Vanhoutte. 1995. Endothelium-dependent hyperpolarization. Beyond nitric oxide and cyclic GMP. Circulation. 92:3337-3349.

4. Shimokawa, H., and A. Takeshita. 1995. Endothelium-dependent regulation of the cardiovascular system. Intern. Med. 34:939-946.

5. Suzuki, H., and G. Cheng. 1990. Endothelium-derived hyperpolarizing factor (EDHF): an endogenous potassium-channel activator. News Physiol. Sci. 5:212-215.

6. Nakashima, M., J.V. Mombouli, A.A. Taylor, and P.M. Vanhoutte. 1993. Endothelium-dependent hyperpolarization caused by bradykinin in human coronary arteries. J. Clin. Invest. 92:2867-2871.

7. Pascoal, I.F., and J.U. Umans. 1996. Effects of pregnancy on mechanisms of relaxation in human omental microvessels. Hypertension (Dallas). 28:183-187.

8. Wallerstedt, S.M., and M. Bodelsson. 1997. Endothelium-dependent relaxation by substance $\mathrm{P}$ in human isolated omental arteries and veins: relative contribution of prostanoids, nitric oxide and hyperpolarization. Br. J. Pharmacol. $120: 25-30$.

9. Shimokawa, H., H. Yasutake, K. Fujii, M.K. Owada, R. Nakaike, Y. Fukumoto, T. Takayanagi, T. Nagao, K. Egashira, M. Fujishima, and A. Takeshita. 1996. The importance of the hyperpolarizing mechanism increases as the vessel size decreases in endothelium-dependent relaxations in rat mesenteric circulation. J. Cardiovasc. Pharmacol. 28:703-711.

10. Rosolowsky, M., and W.B. Campbell. 1993. Role of $\mathrm{PGI}_{2}$ and epoxy- eicosatrienoic acids in relaxation of bovine coronary arteries to arachidonic acid. Am. J. Physiol. 264:H327-H335.

11. Hecker, M., A.T. Bara, J. Bauersachs, and R. Busse. 1994. Characterization of endothelium-derived hyperpolarizing factor as a cytochrome P450-derived arachidonic acids metabolite in mammals. J. Physiol. (Lond.). 481:407-414.

12. Bauersachs, J., M. Hecker, and R. Busse. 1994. Display of the characteristics of endothelium-derived hyperpolarizing factor by a cytochrome $\mathrm{P} 450$ derived arachidonic acid metabolite in the coronary microcirculation. $\mathrm{Br} . \mathrm{J}$. Pharmacol. 113:1548-1553.

13. Najibi, S., C.L. Cowan, J.J. Palacino, and R.A. Cohen. 1994. Enhanced role of potassium channels in relaxation to acetylcholine in hypercholesterolemic rabbit carotid artery. Am. J. Physiol. 266:H2061-H2067.

14. Zygmunt, P.M. 1996. Role of potassium channels in endothelium-dependent relaxation resistant to nitroarginine in the rat hepatic artery. Br. J. Pharmacol. 117:1600-1606.

15. Murphy, M.E., and J.E. Brayden. 1995. Apamin-sensitive $\mathrm{K}^{+}$channels mediate an endothelium-dependent hyperpolarization in rabbit mesenteric arteries. J. Physiol. 489:723-734.

16. Campbell, W.B., D. Gebremedhin, P.F. Pratt, and D.R. Harder. 1996. Identification of epoxyeicosatrienoic acids as endothelium-derived hyperpolarizing factors. Circ. Res. 78:415-423.

17. Mantelli, L., S. Amerini, and F. Ledda. 1995. Role of nitric oxide and endothelium-derived hyperpolarizing factor in vasorelaxant effect of acetylcholine as influenced by aging and hypertension. J. Cardiovasc. Pharmacol. 25: 595-602.

18. Fujii, K., M. Tomonaga, S. Ohmori, K. Kobayashi, I. Koga, Y. Tanaka, and M. Fujishima. 1992. Decreased endothelium-dependent hyperpolarization to acetylcholine in smooth muscle of the mesenteric artery of spontaneously hypertensive rats. Circ. Res. 70:660-669.

19. Cowan, C.L., and R.P. Steffen. 1995. Lysophosphatidylcholine inhibits relaxation of rabbit abdominal aorta mediated by endothelium-derived nitric oxide and endothelium-derived hyperpolarizing factor independent of protein kinase C activation. Arterioscler. Thromb. Vasc. Biol. 15:2290-2297.

20. Eizawa, H., Y. Yui, R. Inoue, K. Kosuga, R. Hattori, T. Aoyama, and S. Sasayama. 1995. Lysophosphatidylcholine inhibits endothelium-dependent hyperpolarization and $N$-nitro-L-arginine/indomethacin-resistant endotheliumdependent relaxation in the porcine coronary artery. Circulation. 92:3520-3526.

21. Najibi, S., and R.A. Cohen. 1995. Enhanced role of $\mathrm{K}^{+}$channels in relaxations of hypercholesterolemic rabbit carotid artery to NO. Am. J. Physiol. 269:H805-H811.

22. Fukao, M., Y. Hattori, M. Kanno, I. Sakuma, and A. Kitabatake. 1996. Endothelium-dependent hyperpolarizations in arteries from diabetic rats. In Endothelium-derived Hyperpolarizing Factor. P.M. Vanhoutte, editor. Harwood Academic Publishers, Amsterdam. 263-270.

23. Fujii, K., S. Ohmori, M. Tominaga, I. Abe, Y. Takata, Y. Ohya, K. Kobayashi, and M. Fujishima. 1993. Age-related changes in endotheliumdependent hyperpolarization in the rat mesenteric artery. Am. J. Physiol. 265: H509-H516.

24. Reinhart, P.H., S. Chung, and I.B. Levitan. 1989. A family of calciumdependent potassium channels from rat brain. Neuron. 2:1031-1041.

25. Canadia, S., M.L. Garcia, and R. Latorre. 1992. Mode of action of iberiotoxin, a potent blocker of the large conductance $\mathrm{Ca}^{2+}$-activated $\mathrm{K}^{+}$channel. Biophys. J. 63:583-590.

26. Urakami, L., H. Shimokawa, M. Nakashima, N. Matsumoto, M.K. Owada, and A. Takeshita. 1997. Relative importance of endothelium-derived hyperpolarizing factor and nitric oxide in coronary arteries in pigs and rabbits. Jpn. J. Pharmacol. 75 (Suppl. I):49P.

27. Weintraub, N.L., S.N. Joshi, C.A. Branch, A.H. Stephenson, R.S. Sprague, and A.J. Lonigro. 1994. Relaxation of porcine coronary artery to bradykinin. Role of arachidonic acid. Hypertension (Dallas). 23:976-981.

28. Corriu, C., M. Feletou, E. Canet, and P.M. Vanhoutte. 1996. Inhibitors of the cytochrome P450-monooxygenase and endothelium-dependent hyperpolarization in the guinea-pig isolated carotid artery. Br. J. Pharmacol. 117:607-610.

29. Tagawa, H., H. Shimokawa, T. Tagawa, M. Kuroiwa-Matsumoto, Y. Hirooka, and A. Takeshita. 1997. Short-term estrogen augments both nitric oxidemediated and non-nitric oxide-mediated endothelium-dependent forearm vasodilation in postmenopausal women. J. Cardiovasc. Pharmacol. 30:481-488. 